\title{
A research on early warning method of Distribution Network Cyber Physical System
}

\author{
Feng Chen ${ }^{1,2,}$, Wei Wei $\mathrm{Xu}^{2}$, Zong Heng Wang ${ }^{1}$, Tao Yang ${ }^{2}$, Hong Yang Huang ${ }^{2}$ \\ ${ }^{1 .}$ Hangzhou E.Energy Technology Co., Ltd, Hangzhou 310000, China \\ 2. State Grid Zhejiang Electric Power Research Institute, Hangzhou 310000, China
}

\begin{abstract}
The high integration of cyber and physics is the development trend of intelligent distribution network in the future, but the cyber system not only supports the stable operation of the physical system, also brings some security risks to the cyber physical system of distribution network. Aiming at the requirements of real-time, accuracy, efficiency and other characteristics of distribution network monitoring, this paper proposes an early warning method of distribution network cyber physical system based on Hidden Markov model. Firstly, the online monitoring and early warning system architecture of distribution network information physical system is proposed, and then the early warning method of distribution network cyber physical system based on Hidden Markov model is established. Finally, an example is given to verify that the proposed strategy can accurately and efficiently early warn the fault.
\end{abstract}

\section{Introduction}

At present, power equipment monitoring is still in the stage of manual inspection ${ }^{[1]}$. However, this method has the following problems: the internal situation of power equipment can not be monitored in real time, a lot of manpower and material resources are consumed in the inspection process, and safety accidents are prone to occur. How to safely and effectively monitor the power equipment in real time and accurately obtain the fault information of the equipment is of great significance to the production and construction of power equipment.

At present, the research of power equipment monitoring at home and abroad mainly focuses on data acquisition and data processing. In reference [2] uses the Internet of things technology to monitor the power equipment, and establishes the electrical equipment monitoring system based on the Internet of things. This technology solves the problem of electrical equipment monitoring, but the system itself does not process the data, the degree of intelligence is not high, and can not carry out real-time warning. In reference [3], the grey GM $(1,1)$ model is used to establish the power equipment prediction model, which realizes the function of fault early warning and greatly improves the early warning fault tolerance rate. However, the model only processes data in the data service layer, and does not involve the construction of data acquisition and visualization platform.

Aiming at the problems existing in the operation and supervision of power equipment which are lack of the above research, this paper innovatively uses the cyber physical system (CPS) and hidden Markov model to build the online monitoring and early warning system of

\footnotetext{
*Corresponding author: zhoudan@zjut.edu.cn
}

distribution network ${ }^{[4]}$. By exploring CPS technologies such as information collection, network transmission and data processing, the system structure constructs a closed-loop system of perception, transmission, analysis, decision-making and execution, realizes the integration of physics and information, improves the overall situation, intelligence and humanization of the system, and realizes the purpose of real-time warning of electric equipment.

\section{Architecture of online monitoring and early warning system for power equipment}

According to the design goal of the system, the CPS technology is used to build an online monitoring and early warning system for power equipment. Combined with the current popular cloud technology, the system is stored in the cloud, and the "cloud" big data storage and comprehensive computing capabilities are used to analyze and process the data in real time. The system architecture can be divided into physical perception layer, network communication layer, data service layer and user application layer. According to the data flow process, the system is divided into global data collection, intelligent data processing and evaluation, and human-computer interaction.

\subsection{Physical perception layer}

The physical sensing layer is composed of various sensors, which can realize the real-time monitoring of power equipment status. The sensors of physical sensing 
layer include temperature sensor, humidity sensor, current sensor, voltage sensor and so on. When the sensing node works, the sensor collects the corresponding data and sends it to the local management unit, and assigns the corresponding time stamp and device number to the data. The data is uploaded to the data server through the communication module. The acquisition of power equipment information in physical perception layer is the basis of communication, calculation and service of power equipment monitoring and early warning system.

\subsection{Network communication layer}

The network communication layer is mainly used for the remote transmission of data, its purpose is to achieve the communication between the various parts of the system, but also to ensure the real-time transmission and reliability. At present, the common wireless transmission methods are WiFi, Lora, 3G / 4G, NB-IOT and so on. Due to the complexity of the internal electrical equipment, the transmission needs to meet the characteristics of high reliability, high speed and strong anti-interference ability.

\subsection{Data service layer}

Data service layer includes two functions: current data storage and historical data reading.

\subsubsection{Process of writing and uploading to cloud server}

(1) Power quality monitoring stations at all levels collect real-time monitoring data through on-site monitoring terminals. At present, file transfer protocol (FTP) is widely used to call PQDIF files.

(2) After the data analysis program in the local server of each monitoring station obtains the original monitoring data, the characteristic values of each index are obtained through the cloud data processing program, and then compressed according to the original data file partition mechanism and written into the local server hard disk, and the file index is formed.

(3) When the system is in the initial state, the power quality index data files of each monitoring point are only stored in the local server of the monitoring station; after the system is put into operation, each monitoring station will upload its stored data information to the cloud server database to ensure the reliability of the monitoring and early warning results of the system.

\subsubsection{Read Historical Data}

(1) The user terminal of a monitoring node directly sends a data query request to the cloud master server, which includes the monitoring point identification code (ID), index type ID and query time span.

(2) After receiving the query request, the master server immediately transmits the index information including the server IP address, file physical address, data starting address and size of the index data file to the terminal. The subsequent reading of this file block is carried out in the local client, so as to reduce the main server's participation in unnecessary communication and improve the delivery efficiency.

(3) The terminal sends a file read request to the corresponding local server, including the file block ID and the starting address of the data in the file block.

(4) After receiving the query request from the user terminal of the node, the local server transmits the historical monitoring data to the terminal through the data transmission channel based on transmission control protocol (TCP).

\section{Data processing and accurate evaluation based on hidden markov model}

\subsection{Overview of hidden markov model}

Hidden Markov model (HMM) is essentially a probability model composed of an invisible state sequence and a general stochastic process which generates some observable sequences from each state. It is generally used to process data with time series relationship between samples, including two random process sequences: state transition sequence (described by state probability) and observation variable sequence (described by probability distribution of observation value). Among them, the state transition sequence is a simple Markov chain, and the observation variable sequence is the sequence corresponding to the state ${ }^{[5]}$.

A complete HMM model generally includes five parameters: state parameter $\mathrm{N}$, observation symbol number $\mathrm{M}$ and three probability density numbers $(\mathbf{A}, \mathbf{B}$, $\pi$ ).

The state parameter $\mathrm{N}$ is the number of states of HMM model, which is the set of hidden states. The set of model states $\mathrm{S}=\left\{\mathrm{S}_{1}, \mathrm{~S}_{2}, \ldots \mathrm{S}_{\mathrm{n}}\right\}, t$ is expressed as $\mathrm{q}_{\mathrm{t}}$, $\mathrm{q}_{\mathrm{t}} \in\left\{\mathrm{S}_{1}, \quad \mathrm{~S}_{2}, \ldots \mathrm{S}_{\mathrm{n}}\right\}$.The hidden state is a Markov process, which describes the actual state of the system.

HMM model observation symbol number set $\mathrm{O}=\left\{\mathrm{O}_{1}\right.$, $\left.\mathrm{O}_{2}, \ldots \mathrm{O}_{\mathrm{M}}\right\}$.

Probability transfer matrix $\mathbf{A}=\left\{\mathrm{a}_{\mathrm{ij}}\right\}$, It is used to indicate that the variable is in state $\mathrm{S}_{\mathrm{i}}$ at time $t$

$$
a_{i j}=P\left(q_{t+1}=S_{j} \mid q_{t}=S_{i}\right), i \geq 1, j \leq N
$$

The matrix $\mathbf{B}$ is the probability matrix that a specific hidden state will produce a specific observation value in a given Markov process. $\mathrm{P}$ is the probability of the system producing the observation value $\mathrm{O}_{\mathrm{k}}$ in the following states

$B=\left\{b_{j}\left(O_{k}\right)\right\}, b_{j}\left(O_{k}\right)=P, 1 \leq j \leq N, 1 \leq k \leq M(2)$

$\pi$ represents the probability distribution of the initial state,

$$
\pi=\left\{\pi_{i}\right\}, \pi_{i}=P\left(q_{1}=S_{i}\right), 1 \leq i \leq N
$$


Using the state transition matrix of hidden Markov model to predict the next most likely state of the system, that is to say, through the calculation of the current state of the system by hidden Markov model, the state of the system at any time can be obtained, and the state transition of the system can be clearly indicated. Although there is a certain time limit, but in a certain period of time, the prediction results are more accurate.

\subsection{Application of hidden markov model}

Taking voltage fluctuation as an example, five hidden Markov transition states are defined according to different frequency of voltage fluctuation. According to the historical data of voltage monitoring, the maximum likelihood estimation method is used to estimate the state transition matrix. Then the generalized autoregressive model of voltage fluctuation index under each state is established, and the least square method is used to estimate the parameters of the regression model Finally, according to the voltage fluctuation index and state transition matrix of $t$ time and $t-1$ time, the predictive value of voltage fluctuation index of each state at $t+1$ time is weighted summation, and the predictive value of voltage fluctuation index at $t+1$ time is obtained.

The development trend of voltage fluctuation with different frequency of voltage fluctuation has different impact on users. According to the frequency of voltage fluctuation, the voltage fluctuation is divided into multiple states, and each state can transfer to each other, and the state of the next moment is only related to the state of the current moment, and has nothing to do with the previous state. Because each voltage fluctuation state is invisible, but each state can obtain a voltage fluctuation index, and the voltage fluctuation index is the probability function of the state, so the voltage fluctuation can be described by hidden Markov model ${ }^{[6]}$.

Referring to the limit value requirements of voltage fluctuation in GB/T 12326-2008, five different hidden transition states of voltage fluctuation are defined.

State $S_{1}$ : voltage fluctuation frequency $r \leq 1$ time $/ \mathrm{h}$, voltage fluctuation index less than $4 \%$.

State $\mathrm{S}_{2}: 1<\mathrm{r} \leq 10$ time $/ \mathrm{h}$, voltage fluctuation index less than $3 \%$.

State $\mathrm{S}_{3}: 10<\mathrm{r} \leq 100$ time/h, voltage fluctuation index less than $2 \%$.

State $\mathrm{S}_{4}: 100<\mathrm{r} \leq 1000$ time/h, voltage fluctuation index less than $1.25 \%$.

State $S_{5}: r \geq 1000$ time $/ h$, voltage fluctuation index is out of limit.

The state of voltage fluctuation at time $t$ is $(t=1$, $2, \ldots), q_{t}=S_{i}$ indicates that the voltage fluctuation is in the state $S_{i}$ at time $t$. In a time series, the system will transfer from one state to another with a certain probability. The transition process is described by the probability transition matrix A between the States. For the first-order Markov model, the state at $t+1$ time is only related to the state at $t$ time, without considering the previous state of the system. The voltage fluctuation state at $t+1$ time is represented by. Then the element in the probability transfer matrix $\mathbf{A}$ is defined as

$$
a_{i j}=P\left(q_{t+1}=S_{j} \mid q_{t}=S_{i}\right)
$$

and met

$$
\sum_{j=1}^{5} a_{i j}=1, a_{i j} \geq 0
$$

$\pi=\left\{\pi_{i}\right\} \quad$ represents the initial probability distribution of the state space

$$
\pi_{i}=P\left(q_{1}=S_{i}\right), i=1,2,3,4,5
$$

According to the state transition results from time 1 to time $t$, the maximum likelihood estimation method is used to estimate the parameters of probability transition matrix A, and the log likelihood equation is obtained as follows

$\frac{\partial \ln L(A)}{\partial A}=\frac{\partial\left(\sum_{i=1}^{t-1} \ln P\left(q_{i+1} \mid q_{i}\right)+\ln P\left(q_{1}\right)\right)}{\partial A}=0$

Where: $L(A)$ is the likelihood function of probability transfer matrix; $q_{i}$ is the voltage fluctuation state at time $\mathrm{i} ; q_{i+1}$ is the voltage fluctuation state at time $\mathrm{i}+1$.

By observing the voltage fluctuation index at time $t$, the observed value $y_{t}$ is related to the state of voltage fluctuation at time $t$. Considering the influence of voltage fluctuation index at $t-1$ time and $t$ time on voltage fluctuation index at $t+1$ time, a generalized autoregressive model of voltage fluctuation index at each state is established, which is the hidden Markov transformation model of voltage fluctuation.

$$
y_{t+1}^{i}=u_{i}+\beta_{i} y_{t}+\alpha_{i} y_{t-1}+u_{t}^{i}
$$

$y_{t}, y_{t-1}$ are voltage fluctuation index at time $t$ and time $t-1 ; y_{t+1}^{i}$ is in state $S_{i}$, prediction value of voltage fluctuation index at time $t+1$, parameter $u_{i}, \beta_{i}, \alpha_{i}$ are regression coefficient in state $S_{i}$, the least square method is used to estimate the parameters. $u_{t}^{i}$ is white noise in state $S_{i}$, obey $N\left(0, \sigma_{i}^{2}\right)$.

According to the voltage fluctuation index at $t$ time and $t-1$ time, the predictive value of voltage fluctuation index at $t+1$ time in each state is obtained. Then, according to the state transition matrix $\mathbf{A}$, the predictive value of voltage fluctuation index at $t+1$ time in each state is weighted and summed to obtain the predictive value of voltage fluctuation index at $t+1$ time Measured value, i.e

$$
y_{t+1}=\sum_{j=1}^{5} a_{i j} y_{t+1}^{i}=\sum_{j=1}^{5} a_{i j}\left(u_{i}+\beta_{i} y_{t}+\alpha_{i} y_{t-1}+u_{t}^{i}\right)
$$




\section{Case analysis}

The early warning system has been applied in a certain area, and the voltage fluctuation that may occur during its operation is predicted and analyzed, as shown in Figure 1.

The voltage fluctuation prediction curve is shown in Figure 4a. Except that the voltage fluctuation of phase $\mathrm{C}$ exceeds the limit value at 23:10, it does not exceed the limit value in other time periods. According to the actual voltage fluctuation curve in the same period, as shown in Figure $1 \mathrm{~b}$, the voltage fluctuation does exceed the limit at about 23:10, which proves that the early warning method of distribution network information physical system based on Hidden Markov model proposed in this paper has certain accuracy in monitoring and forecasting
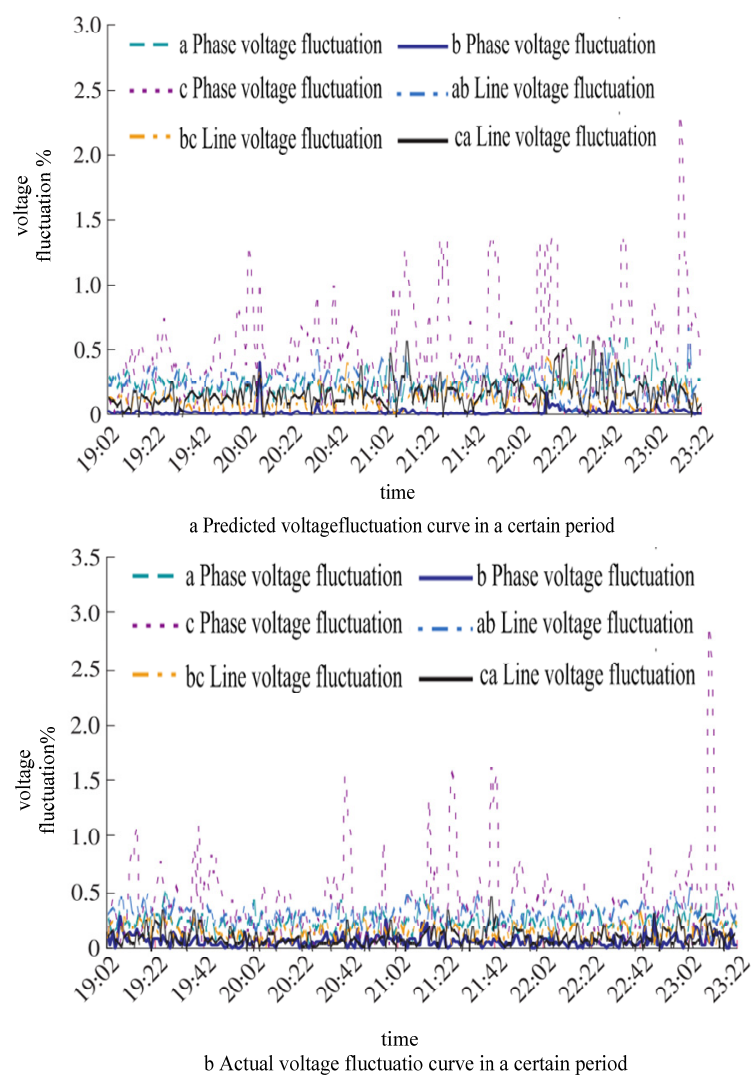

Figure 1 Comparison of voltage fluctuation prediction and actual fluctuation curve in a certain period

\section{Conclusion}

In this paper, an online detection and early warning system of power equipment based on CPS is constructed. Using CPS technology, fault early warning is carried out on the basis of power quality index prediction method based on Hidden Markov model. In the actual distribution network practice, good prediction results are obtained, which proves the feasibility of the method, and provides an effective and reasonable solution for the early warning of distribution network in reality.

\section{Reference}

1. Li Y ,Chen M , Dai W , et al. Energy Optimization With Dynamic Task Scheduling Mobile Cloud Computing[J]. IEEE Systems Journal, 2017,PP(1):1-10.

2. Ganga, D, Ramachandran, et al. IoT-Based Vibration Analytics of Electrical Machines[J]. IEEE Internet of Things Journal, 2018.

3. Tian D , Xiong X, Xiao C. Early Warning and Inhibition of HVDC Subsequent Commutation Failure during Recovery Process under Grid Fault[J]. IEEE Transactions on Power Delivery, 2020, PP(99):1-1.

4. Wills R F .Development of a Cyber Physical System for Fire Safety[J]. Dissertations \& Theses Gradworks, 2016.

5. Shinji, Watanabe, Atsushi, et al. Structural Bayesian Linear Regression for Hidden Markov Models[J]. Journal of Signal Processing Systems, 2014.

6. MO Huadong, SANSAVINI Giovanni. Hidden Markov Model-based Smith predictor for the mitigation of the impact of communication delays in wide-area power systems [J]. Applied Mathematical Modelling, 2021, 89 (Part 1): 19-48. 